\title{
EDITORIAL
}

\section{Discriminación algorítmica y derecho granular: nuevos retos para la igualdad en la era del big data}

\author{
Algorithmic discrimination and granular law: new challenges \\ for equality in the age of big data
}

Jesús R. Mercader Uguina*

Catedrático Derecho del Trabajo y la Seguridad Social

Universidad Carlos III de Madrid

ORCID ID: 0000-0001-7153-0701

doi: https://doi.org/10.20318/labos.2021.6213

"La ciencia no piensa"

M. Heidegger, ¿Qué significa pensar?

\section{Big data, algoritmos y sentimientos humanos}

Estamos en la era del Big Data. El rápido desarrollo de la economía de los datos y de las tecnologías emergentes, como la inteligencia artificial, productos y servicios del «internet de las cosas», sistemas autónomos y la tecnología 5G, están planteando nuevos problemas jurídicos tras los cuales se sitúa el debate sobre las limitaciones en el uso de los datos y, muy en particular, de los personales. Estas tecnologías están conduciendo a la consideración de los datos digitales como materia prima capital de la sociedad de la información y del conocimiento, el aire que respira esta nueva realidad. Y es que, a medida que la transformación digital se expande hacia más y más áreas de la vida, los modelos de negocio que se desarrollan se basan, precisamente, en el análisis de esos datos lo que, inevitablemente se proyecta sobre lo laboral y, más ampliamente, sobre la legislación social en su conjunto.

Hasta ahora, el interés por estos análisis había prestado atención a los potenciales peligros y riesgos para la privacidad y la autonomía de los individuos y, sobre esta base, se centraba en la elaboración de marcos de regulación que limitasen el uso y tratamiento de los datos personales. Sin embargo, la transformación digital va a exigir ir más allá y va a requerir también una reflexión muy profunda sobre las diferentes perspectivas y riesgos que pueden llevar consigo estas técnicas. Todo ello en la medida en que el uso del Big Data está permitiendo reelaborar gran cantidad de datos simples, de forma que, combinados entre sí, puede contribuir a efectuar la más precisa y exacta

*jesus.mercader@uc3m.es 
individualización de una persona alcanzando niveles máximos de granularidad o, si se prefiere, de especificidad. El auge de la sociedad basada en los datos podría cambiar la forma en que se diseñan las normas. La idea de un Derecho también granular, esto es, de un Derecho adaptado a las concretas y particulares circunstancias de cada persona no es, como veremos seguidamente, nada remota.

A este análisis no resulta ajeno el desarrollo de los algoritmos definidos como conjuntos ordenados y finitos de operaciones que permiten hallar la solución de un problema. Una tecnología intelectual, se ha dicho, que supone, en última instancia, la sustitución de juicios intuitivos por una respuesta objetivada. Y ello en la medida en que un algoritmo es una receta, un conjunto de instrucciones matemáticas, una secuencia de tareas destinada a conseguir un cálculo o un resultado. En suma, los algoritmos contribuyen a formalizar una serie de reglas de decisión y a efectuar cadenas de cálculos que permiten el análisis de múltiples variables seleccionando, de entre ellas, la mejor. Una realidad lejana y difícilmente comprensible para los juristas, quienes, por inercia natural, miramos con desconfianza la esférica perfección de las matemáticas.

Una desconfianza que tiene su fundamento. Se ha dicho que las estadísticas, cuya proyección en la gestión del Big Data es esencial, son como los presos: con la suficiente mala intención se les puede torturar hasta que confiesen. $Y$ es que las informaciones estadísticas, aunque se nos aparecen como hechos fríos, sólidos, realidades reveladas que nos ofrece la naturaleza, y por ello incuestionables, son, en última instancia, resultado del actuar humano. Pero, como dice Levitin en un libro cuyo título es revelador ("La mentira como arma", Alianza Universidad, 2019), es importante recordar que quienes elaboran (reténgase la noción) las estadísticas son personas. Y son, precisamente, éstas las que deciden qué contabilizar, cómo hacerlo y qué parte de las cifras resultantes van a compartir con nosotros. En suma, concluye Levitin, "las estadísticas no son hechos. Son interpretaciones".

El cruce de las anteriores lógicas irradia sus efectos en múltiples campos del actuar humano y, como no podía ser de otro modo, también en el laboral. Aquí, es la empresa la que ha incorporado su uso hasta el punto de que parece que el empresario está dispuesto a delegar o, si se prefiere, a descentralizar parte de sus poderes tradicionales trasladando un importante número de decisiones a la presunta objetividad y plena fiabilidad que proporciona el recurso al Big Data. Y ello en la medida en que su uso actual se proyecta sobre prácticamente la totalidad de las facetas que componen su autonomía organizativa, recorriendo transversalmente la libertad de actuación empresarial, abarcando desde la selección de trabajadores hasta la forma y modo de ejercicio del poder de dirección, incluyendo sus dimensiones disciplinarias. Un vasto territorio que puede quedar, a no tardar, anegado por las plurales dimensiones y usos que permite la Inteligencia Artificial ("IA").

\section{Tratamientos automatizados de datos y diseńo de perfiles: La sombra de la discriminación algorítmica}

Pero lo cierto es que, aunque los ingenieros de software definen parámetros de análisis de minería de datos, los valores humanos están incrustados en cada paso en su diseño, por lo que el establecimiento de sistemas automatizados de decisión y los datos que les sirven de base pueden encontrarse sesgados en su origen, con lo que su tratamiento debe encontrarse sometido a importantes cautelas. El art. 22.1 RGPD sienta, por ello, una prohibición general de las decisiones basadas únicamente en el tratamiento automatizado, entendiendo por tal, "toda forma de tratamiento automatizado de datos personales consistente en utilizar datos personales para evaluar determinados aspectos personales de una persona física, en particular para analizar o predecir aspectos relativos al rendimiento profesional, situación económica, salud, preferencias personales, intereses, fiabilidad, comportamiento, ubicación o movimientos de dicha persona física" (art. 4.4 RGPD). 
Se establece, de este modo, "el derecho a no ser objeto de una decisión basada únicamente en el tratamiento automatizado, incluida la elaboración de perfiles, que produzca efectos jurídicos en él o le afecte significativamente de modo similar". Ahora bien, también contempla una serie de excepciones (art. 22.2 RGPD) entre las que se encuentran que sea necesario para la conclusión o ejecución de un contrato entre el interesado y un responsable del tratamiento, o en los casos en los que el interesado haya dado su consentimiento explícito. Excepciones que dejan un amplio espacio a estas técnicas en lo laboral. En todo caso, como precisa el Considerando (63) del RGPD, "todo interesado debe, por tanto, tener el derecho a conocer y a que se le comuniquen, en particular, los fines para los que se tratan los datos personales, su plazo de tratamiento, sus destinatarios, la lógica implícita en todo tratamiento automático de datos personales y, por lo menos cuando se base en la elaboración de perfiles, las consecuencias de dicho tratamiento".

De los riesgos de estas técnicas es consciente el WP251 rev.01 del GT29 (actual, Comité Europeo de Protección de Datos), que en sus "Directrices sobre decisiones individuales automatizadas y elaboración de perfiles a los efectos del Reglamento 2016/679” (“WP 251 del GT29”), subraya el peligro asociado al uso de estas fórmulas señalando que: "la elaboración de perfiles y las decisiones automatizadas pueden plantear riesgos importantes para los derechos y libertades de las personas que requieren unas garantías adecuadas. Estos procesos pueden ser opacos. (...) La elaboración de perfiles puede perpetuar los estereotipos existentes y la segregación social. Asimismo, puede encasillar a una persona en una categoría específica y limitarla a las preferencias que se le sugieren (...) En algunos casos, la elaboración de perfiles puede llevar a (...) una discriminación injustificada”.

$\mathrm{Y}$ es que, a diferencia de las personas, los algoritmos son en su esencia sumamente precisos y ciegos a las emociones. Por sí solos no crean nada ni cumplen más tareas que aquellas que se les encargan. Son capaces de hacer operaciones de análisis matemático, pero no prever relaciones ni verdades analíticas. Como decía AlanTuring, no pueden sorprendernos. Somos los seres humanos los que les insuflamos vida y, a su través, sentimientos lo que hace que pierdan su fría lógica y, por tanto, se humanizan y pueden también, como los humanos, discriminar. Ello ocurre porque el aire que respiran los algoritmos, los datos, puede encontrarse viciado por lo que las decisiones automatizadas resultantes se encontrarán, igualmente, corrompidas (sesgos, discriminación, etc.). Por ello, un importante problema al que se enfrenta esta realidad es la "discriminación algorítmica" entendiendo por tal la que se produce cuando un individuo o colectivo recibe un tratamiento arbitrario como consecuencia de la toma de decisiones automatizadas. Una discriminación que obtiene, por el momento, respuestas desde nuestras actuales técnicas conceptuales pero que requeriría probablemente también de nuevas herramientas jurídicas de análisis. La discriminación afronta, pues, nuevos retos.

La discriminación algorítmica en el acceso al empleo comienza a ser una realidad y los derechos de los interesados deben contar con garantías reforzadas en estos casos. De ella hemos hablado en trabajos anteriores en los que hemos valorado los riesgos y efectos del uso de algoritmos en los procesos de selección ("Algoritmos: personas y números en el Derecho Digital del trabajo", Diario La Ley, No 48, Sección Ciberderecho, 24 de febrero de 202). Así lo ha venido a subrayar el apartado VIII de la Carta de Derechos Digitales donde expresamente se señala que: "Se fomentará que los procesos de transformación digital apliquen la perspectiva de género adoptando, en su caso, medidas específicas para garantizar la ausencia de sesgos de género en los datos y algoritmos usados".

No debe sorprender que se llame, por ello, a una intervención efectiva de los representantes de los trabajadores en estos casos. En este sentido, el Dictamen del Comité Económico y Social Europeo "Generar confianza en la inteligencia artificial centrada en el ser humano» ha recordado "la necesidad de consultar e informar a los trabajadores y sus representantes a la hora de introducir sistemas de IA que pudieran provocar cambios en la organización del trabajo, la vigilancia y su control, así como en los sistemas de evaluación y contratación de los trabajadores". Una primera 
materialización de esta idea la podemos encontrar en nuestro país en el Real Decreto-ley 9/2021, de 11 de mayo, por el que se modifica el texto refundido de la Ley del Estatuto de los Trabajadores, aprobado por el Real Decreto Legislativo 2/2015, de 23 de octubre, para garantizar los derechos laborales de las personas dedicadas al reparto en el ámbito de plataformas digitales, que incorpora a la normativa vigente la extensión de los derechos de información de los representantes legales de los trabajadores a aquellas situaciones en las que se implanten sistemas de organización y control del trabajo cuando los mismos incorporen algoritmos o criterios algorítmicos en su realización. Una línea que ha recogido también la citada Carta de Derechos Digitales (apartado XIX) e, incluso, la propia negociación colectiva de la que es buen ejemplo el XXIV Convenio colectivo del sector de la banca $Y$ es que, si resulta exigible normativamente la existencia de una información significativa sobre la «lógica aplicada» al uso de estas herramientas matemáticas, es imprescindible también el desarrollo de instrumentos que materialicen el derecho a la transparencia: un auténtico derecho fundamental de la nueva ciudadanía digital.

Pero la discriminación algorítmica aparece también en el desarrollo de la propia actividad laboral. La delegación del empresario de buena parte de sus decisiones en los algoritmos resulta especialmente trascedente en las nuevas formas de trabajo. Y es que las plataformas profesionales basan su actuación en algoritmos y, a través de los mismos, efectúan asignaciones de actividades a los profesionales incluidos dentro de las mismas. Y es aquí donde aparece la nueva "discriminación algorítmica en el empleo". Buen ejemplo es la reciente sentencia del Tribunale Ordinario di Bologna que, en su sentencia de 31 de diciembre de 2020 (N. R.G. 2949/2019) ha declarado discriminatorio el algoritmo Frank utilizado por Deliveroo en su plataforma online para clasificar o definir el "ranking reputacional" de los riders.

Según quedó acreditado, los repartidores gozan de dos vías para recibir encargos de viajes por parte de la empresa: pueden reservar sesiones con antelación a través del sistema de reserva SSB (“Self-Service Booking”) o pueden iniciar sesión en tiempo real. El sistema de reserva SSB proporciona a los riders un calendario de disponibilidad de la semana entrante para poder recibir encargos de viaje de acuerdo con un ranking (una clasificación) establecido. Los parámetros de dicha clasificación son la llamada "tasa de fiabilidad" (número de veces en el que el rider no atendió una sesión que previamente reservó) y la "tasa de participación en los picos" (número de veces en que el rider estuvo disponible para los horarios más relevantes, es decir, de las $20 \mathrm{~h}$ a las $22 \mathrm{~h}$ de viernes a domingo). Sobre esta base la empresa utiliza un algoritmo que, a juicio del referido Tribunal, penaliza de igual forma y sin distinción alguna tanto a los riders que se ausentan temporalmente por motivos injustificados como a los que lo hacen por motivos justificados enfermedad, cuidado de menores o para ejercer su derecho de huelga. Tal diferencia, concluye el Tribunal, encubre una discriminación algorítmica en el empleo. Y llega a esta conclusión indicando que es indiferente que a los repartidores se les considere trabajadores por cuenta ajena o trabajadores autónomos, dado que en cualquiera de los casos estarán protegidos frente a la discriminación en el acceso al trabajo.

El control sobre los análisis y algoritmos (y especialmente Big Data) es complejo por varias razones. En primer lugar, el estatus profesional de quienes trabajan en el campo es incierto, no hay calificaciones obligatorias o ética profesional compartida. En segundo lugar, la comunidad de "creadores de algoritmos" pertenece casi en su totalidad al sector privado que es el principal consumidor de estos productos. Esta comunidad es global y constantemente interconectada: soluciones desarrolladas en Shanghái en unas pocas semanas podría ser utilizadas por otra persona en Hamburgo o San Francisco (V. Zeno-Zencovich, "Smart contract", "Granular Norms" and Non-Discrimination, en Busch/De Franceschi, Algorthimic Regulation and Personalized Law, Munich, Verlag CH Beck, 2021, p.277).

Si bien el desarrollo de los sistemas de Big Data es apropiado en el funcionamiento de una empresa privada en la medida en que las mismas pagan por sus propios errores, más dudas se plantean 
cuando tales productos digitales deben perseguir políticas públicas. Ejemplo de ello es el algoritmo SyRI (System Risk Indication) que fue utilizado por autoridades públicas para predecir la probabilidad de fraude a la Seguridad Social y al fisco por parte de los solicitantes de beneficios estatales. El Tribunal de lo Civil de la Haya, en sentencia de 5 de febrero de 2020, indicó que existe una responsabilidad especial en el uso de las tecnologías emergentes y concluye que el empleo de SyRI supone un incumplimiento del artículo 8 del Convenio Europeo de los Derechos Humanos (el derecho al respeto de la vida privada y familiar) debido a la falta de transparencia y uso sesgado de este instrumento, utilizado exclusivamente en barrios donde viven personas con rentas bajas o zonas donde residen personas pertenecientes a minorías. Una sentencia que recuerda la STJUE (Gran Sala) de 16 de julio de 2015 en el asunto C-83/14, Chez Razpredelenie Bulgaria AD, en la que se debatía, precisamente, sobre la proporcionalidad de una medida que suponía un perjuicio excesivo para un grupo étnico que se veía estigmatizado sobre la base de consideraciones puramente económicas.

\section{III. ¿¿Normas granulares?: ¿¿Hacia un Derecho personalizado?}

Pero el desarrollo del Big Data afecta a la igualdad también desde una distinta perspectiva: su potencial desarrollo como instrumento de personalización e individualización de las normas. Es evidente que los sistemas legales han crecido granular y gradualmente a través de la jurisprudencia, es decir, por medio de decisiones establecidas caso por caso sobre la base de los hechos, las cualidades y la relación entre las partes en cada concreto y particular supuesto. La sentencia pulveriza la regla general en sus miles de posibles situaciones y aplica las normas casuísticamente. Pero los ejemplos se multiplican. Cuando una empresa cotiza a la Seguridad Social o se solicita una pensión de jubilación con la ayuda de un software público se observa una forma de "normativa granular". En estos casos, hay un marco legal de referencia, pero la cantidad por la que la empresa debe cotizar o la pensión que el trabajador debe recibir depende de la información proporcionada o ya disponible para el organismo público y la "clase" a la que pertenece el individuo. La norma está incorporada en el software que establece de forma univoca las exigencias que cada individuo debe cumplir. En estos casos, no hay apenas margen de error porque el software no permite completar el procedimiento si detecta desviación de la regla.

Esta proyección de la "sociedad de singularidades", como la califica Reckwitz, resulta sorprendente para cualquier jurista y ello porque las transformaciones operadas en la definición del Estado de Derecho desde finales del XIX habían servido para construir un sistema normativo caracterizado por la generalidad de las normas y su abstracción. Sin duda por ello se ha dicho que la estabilidad, la permanencia y la generalidad son características esenciales de la Ley o, más gráficamente expresado, que "la ley es expresión de la voluntad general acatada por todos, configurando el mundo de la seguridad en el que el Derecho fija reglas de juego generales y abstractas que serán utilizadas por cualquier persona en cualquier circunstancia" (J. A. Montilla Martos, Las leyes singulares en el ordenamiento constitucional español, Madrid, Civitas, 1994). En este contexto, la idea de "normas granulares" no dejaría de ser un oxímoron ya que legislar significa generalizar.

Una herramienta para generalizar comúnmente utilizada por el legislador son las que podríamos denominar "tipificaciones" (en expresión de C. Busch, Algorithmic Regulation and (Im) Perfect Enforcement in the Personalized Economy. en Busch/De Franceschi, Algorthimic Regulation and Personalized Law, Munich, Verlag CH Beck, 2021). Estos son modelos normativos que dividen las infinitas variaciones del mundo social en ciertas categorías que crean un orden significativo. Un ejemplo clásico es la determinación de capacidad por referencia a una determinada edad lo que evita hacer indagaciones concretas sobre la facultad cognitiva y madurez de una persona. Las normas 
sobre capacidad jurídica no tienen en cuenta la madurez real y juicio de un específico individuo, no miden la aptitud de cada persona. Esta individualización ha sido vista hasta ahora como arbitraria e insegura. Lo mismo ocurre en materia de derecho de daños cuando se establecen criterios de imputación objetivos o culpabilísticos generalizados. En estos casos, estas aproximaciones legales pueden conducir a errores de regulación y desigualdades. En tan incierto escenario, las reglas legales personalizadas o "granulares" a través de fórmulas de Big Data podrían tener en cuenta la heterogeneidad de los actores en un grado que las leyes generales y abstractas no pueden contemplar.

Igualmente, Big Data e IA pueden contribuir también a individualizar los estándares legales de conducta presentes en muchas normas. Así, se entiende que una norma tiene carácter de estándar cuando tiende a llevar al intérprete a la interpretación directa del principio o valor subyacente a una concreta situación. De este modo, los estándares rebajan el margen de error de las decisiones otorgando al intérprete la posibilidad de tomar en consideración todos los factores relevantes de la totalidad de las circunstancias a través de un proceso de ponderación entre los distintos intereses en juego. En el caso, por ejemplo, del estándar de "persona razonable" no hay precepto que defina lo que es razonable y lo que no lo sería. La granularidad permitiría construir lo que Porat llama el estándar de "usted razonable" (citado por C. Busch \& A. De Franceschi, Granular Legal Norms: Big Data and the Personalization of Private Law, en V. Mak, E. Tjong Tjin Tai y A. Berlee, Research Handbook in Data Science and Law Research Handbooks in Information Law, 2018), un nivel personalizado de diligencia que se requiere a cada actor individual para cumplir con un estándar de atención personalizado basado en la información sobre sus concretas e individuales características. Ello permitiría resolver lo que Oliver Wendell Holmes llamó a la "ecuación personal” de cada sujeto. Obviamente, modelos de estas características siembran sombras de duda sobre potencial el grado de intervención sobre la privacidad que sería necesaria para alcanzar tan exacta valoración.

Algunos autores [A. J. Casey \& A. Niblett, "The Death of Rules and Standards" (Coase-Sandor Working Paper Series in Law and Economics, 2015, no 738; (uchicago.edu)] llevan la idea del derecho personalizado incluso un paso más allá y prevén un escenario en el que los avances tecnológicos podrían permitir a los legisladores promulgar leyes complejas a través de micro-directivas. Estas micro-directivas se adaptarían con precisión a todos los escenarios posibles y proporcionarán instrucciones claras en tiempo real (posiblemente a través de asistentes digitales personales o mediante realidad aumentada) sobre cómo cumplir con la ley. Lo cierto es que la idea de granularidad normativa vuela por los aires los cimientos del modelo clásico de Estado de Derecho en la medida en que el desarrollo de los sistemas de Big Data podría permitir personalizar la ley y lograr un nivel de hiperindividualización que hasta ahora no se ha conseguido, lo que llevaría consigo una transformación radical del equilibrio entre la equidad individual y la seguridad jurídica.

Probablemente, antes de llegar a ese resultado el Big Data podría convertirse en un instrumento sino de transformación si de modulación del alcance de las normas jurídicas. Las nuevas técnicas pueden servir para precisar los supuestos de lógica borrosa presentes en muchas disposiciones. Es ésta una lógica divergente, alejada de los modelos lógicos binarios clásicos que habían venido siendo aplicados al Derecho sobre la base del carácter cerrado del ordenamiento jurídico. Como explica B. Kosko, la mayoría de los conceptos son borrosos porque tienen fronteras imprecisas. No existen líneas de trazo definido entre el agua que está caliente y la que no lo está. Nuestras ideas son intrínsecamente borrosas y nuestro razonamiento aproximado. En el Derecho sucede algo parecido, la realidad ofrece fronteras difusas en la construcción y en el diseńo de las normas. Los sistemas de IA pueden contribuir decisivamente a dar respuestas a realidades normativas borrosas. Como se ha dicho, la lógica borrosa es un instrumento adecuado para captar y detectar algunos rasgos destacados del actuar jurídico en situaciones de incertidumbre (T. Mazzarese, Lógica borrosa y decisiones judiciales: el peligro de una falacia racionalista, Doxa, 1996, no 19). 
Las propuestas para que la lógica borrosa se proyecte en el terreno de la protección social no son nuevas. En uno de los libros más inteligentes y brillantes que se han escrito en nuestro país en esta materia, "Análisis crítico del sistema español de pensiones no contributivas", de Josep Fargas Fernández, ya se apunta esta idea. En él se dice: "En el Derecho de la Seguridad Social creemos que el uso de la lógica borrosa puede resultar útil (...), a un discapacitado con un $64 \%$ de invalidez no se le proporcionará una prestación, y cuando alcance el $65 \%$ de minusvalía podrá causar una pensión vitalicia de invalidez. Si observamos los distintos requisitos que deben cumplirse para causar una pensión de invalidez no contributiva observaremos que la mayoría de ellos se trata de parámetros que son progresivos: edad, grado de invalidez, duración de la residencia previa, carencia de rentas (...) Tales cálculos requieren una investigación previa multidisciplinar (...). Asimismo, dichos cálculos requerirán el uso de computadoras dada la complejidad de las interrelaciones (intersecciones y exclusiones) entre los distintos factores (...), lo que aquí se propone es (...) aproximar las prestaciones a la necesidad que se va a tutelar".

La precisión del derecho personalizado depende de la calidad y cantidad de datos que alimentan los sistemas de IA y el riesgo de las discriminaciones algorítmicas es una realidad cierta. Por ello parece claro que el Derecho camina por la senda de la tecnología y todo ello nos exige repensar con nueva mirada el sistema jurídico en su conjunto y, muy en particular, el que se proyecta sobre el universo normativo de lo social. 